\title{
Atividades biológicas de espécies amazônicas de Rubiaceae
}

\begin{abstract}
MESQUITA, D.W.O.1,2,3* MESQUITA, A.S.S. C.C.S. ${ }^{5}$; NOVAES, J.A.P. ${ }^{1}$; OLIVEIRA, J.A.A. ${ }^{6}$; NUNEZ, C.V. ${ }^{1}$.

'Laboratório de Bioprospecção e Biotecnologia - LABB, Coordenação de Tecnologia e Inovação, Instituto Nacional de Pesquisas da Amazônia (INPA), Av. André Araújo, 2936, Aleixo, Manaus, AM, Brasil, CEP 69060-001, CP 478. 2Departamento de Engenharia de Produção, Campus de Cacoal, Universidade Federal de Rondônia (UNIR), Rua da Universidade, 920, Jardim São Pedro II, Cacoal, RO, Brasil, CEP 76962-384. ${ }^{3 P r o g r a m a}$ de Pós-Graduação em Química, Universidade Federal do Amazonas (UFAM), Av. Gal. Rodrigo Octávio, 6.200, Coroado I, Manaus, AM, Brasil, CEP 69060-001. 'Universidade Estadual de Roraima (UERR) - Campus Rorainópolis, Av. Senador Hélio Campos, s/n, Centro, Rorainópolis, RR, Brasil, CEP 69373-000. ${ }^{5}$ Laboratório de Farmacologia, Instituto Nacional de Pesquisas da Amazônia (INPA), Av. André Araújo, 2936, Aleixo, Manaus, AM, Brasil, CEP 69067375, CP 478. ${ }^{6}$ Laboratório de Micologia, Instituto Nacional de Pesquisas da Amazônia (INPA), Av. André Araújo, 2936, Aleixo, Manaus, AM, Brasil, CEP 69060-001, CP 478. *Autor para correspondência: dennymesquita@ yahoo.com.br
\end{abstract}

RESUMO: Rubiaceae é uma família botânica com grande potencial químico e biológico Neste trabalho realizou-se a prospecção fitoquímica e avaliou-se as atividades citotóxica, antioxidante e antifúngica dos extratos diclorometânicos e metanólicos das folhas de Duroia saccifera, Ferdinandusa goudotiana, F. hirsuta, F. paraensis, Ferdinandusa sp., Palicourea corymbifera e $P$. guianensis. A avaliação da citotoxicidade foi realizada empregando-se o ensaio de toxicidade sobre Artemia salina. A atividade antifúngica foi determinada pelo método de difusão em ágar utilizando-se os fungos: Epidermophyton floccosum, Microsporum canis 32905, M. gipseum 29/00, Trichophyton mentagrophytes ATCC 9533/03, T. rubrum ATCC 28189, T. tonsurans 21/97, Cladosporium cladosporioides, C. sphaerospermum, Fusarium U. 662/06, Scytalidium U. 661/06, Candida albicans ATCC 3632 e C. albicans U. 5/99. A atividade antioxidante foi determinada através dos ensaios de redução do radical livre 1,1-difenil-2-picrilhidrazila (DPPH) e de descoloração do cátion radical ácido-6-sulfônico-2,2-azinobis-3-etilbenzotiazolina (ABTS). Para o ensaio de toxicidade sobre $A$. salina observou-se maior toxicidade no extrato metanólico de $F$. goudotiana, que se mostrou tóxico até a concentração de $5 \mu \mathrm{g} \mathrm{mL} \mathrm{L}^{-1}$. A presença de princípios antifúngicos foi observada em $F$. hirsuta e $F$. paraensis contra quatro dos fungos testados. $\mathrm{Na}$ avaliação da atividade antioxidante os extratos metanólicos mostraram maior atividade que os extratos diclorometânicos, sendo coincidente o resultado obtido através de ambos os métodos (redução do DPPH e descoloração do ABTS). Os resultados criam possibilidades para futuras investigações relacionadas à estrutura e atividade dos componentes de cada extrato ativo.

Palavras-chave: Artemia salina, Antioxidante, Antifúngica, Duroia, Palicourea.

ABSTRACT: Biological activities of Amazonian species of Rubiaceae. Rubiaceae is a plant family with great chemical and biological potential. In this work a phytochemical prospection was performed and it was assessed the cytotoxic, antioxidant and antifungal activities of dichloromethanic and methanolic extracts of the leaves of Duroia saccifera, Ferdinandusa goudotiana, F. hirsuta, F. paraensis, Ferdinandusa sp., Palicourea corymbifera and P. guianensis. The cytotoxicity evaluation was carried out using the test toxicity on Artemia salina. The antifungal activity was determined by agar diffusion method using fungi: Epidermophyton floccosum, Microsporum canis 32905, M. gipseum 29/00, Trichophyton mentagrophytes ATCC 9533/03, T. rubrum ATCC 28189, T. tonsurans 21/97, Cladosporium cladosporioides, C. sphaerospermum, Fusarium U. 662/06, Scytalidium U. 661/06, Candida albicans ATCC 3632 and C. albicans U. $5 / 99$. The antioxidant activity was determined by testing the amount of free radical 1,1-diphenyl2-picrylhydrazyl (DPPH) and the discoloration of the radical cation 6-sulfonic acid-2,2-azinobis3-ethylbenzothiazoline (ABTS). For the toxicity test on $A$. salina greater toxicity in the methanol

Recebido para publicação em 14/11/2012

Aceito para publicação em 06/10/2014

$10.1590 / 1983-084 X / 12 \_153$

Rev. Bras. PI. Med., Campinas, v.17, n.4, p.604-613, 2015. 
extract of $F$. goudotiana was observed, which proved to be toxic up to a concentration of $5 \mu \mathrm{g}$ $\mathrm{mL}^{-1}$. The presence of antifungal principles was observed in the $F$. hirsuta and $F$. paraensis against four tested fungi. In the evaluation of the antioxidant activity, the methanol extracts showed greater activity than the dichloromethanic ones, being similar the obtained result through both methods (reduction of DDPH and ABTS decolorization). The results create possibilities for future researches related to the structure and activity of the components of each active extract.

\section{Keywords: Brine shrimp, Antioxidant, Antifungic, Duroia, Palicourea.}

\section{INTRODUÇÃO}

A Amazônia é possuidora de uma imensa biodiversidade bem pouco explorada, neste contexto destaca-se a família Rubiaceae Juss., conhecida por sua importância medicinal, econômica e ornamental, além da potencialidade tóxica de algumas de suas espécies (Ribeiro et al., 1999), que têm sido estudadas ao longo dos anos (Souza et al., 2013; Vieira et al., 2001), mais especificamente suas espécies amazônicas (Valente et al. 2009; Cardoso et al. 2008; Martins et al., 2013). Devido à importância dessa família resolveu-se investigar o potencial de três diferentes gêneros: Duroia L. f., Ferdinandusa Pohl e Palicourea Aubl. As espécies selecionadas foram Duroia saccifera (Mart. ex Roem. \& Schult.) Hook. F. ex Schum., Ferdinandusa goudotiana K. Schum., F. hirsuta Standl., F. paraensis Ducke, Ferdinandusa sp., Palicourea corymbifera (Müll. Arg.) Standl. e $P$. guianensis Aubl. Destas, apenas $P$. corymbifera e $P$. guianensis apresentam registro de estudo na literatura consultada, sendo utilizadas no tratamento de tosses persistentes, e como vermífuga, respectivamente, e não apresentaram letalidade sobre Artemia franciscana (Quignard et al., 2003). Também foi encontrado que extratos liofilizados de $P$. corymbifera não mostraram toxicidade para ratos de laboratório (Assis et al., 2006). As demais espécies não apresentam registros de estudos químico e/ ou biológico na literatura consultada. Objetivou-se com este trabalho realizar uma triagem de todos os extratos sobre as atividades citotóxica sobre Artemia salina, antioxidante e antifúngica e ainda realizar a prospecção fitoquímica dos mesmos. Os ensaios biológicos realizados levam em consideração a simplicidade, a rapidez, a reprodutibilidade e o baixo custo.

\section{MATERIAL E MÉTODOS Plantas e Extratos}

Os extratos diclometânicos e metanólicos de Ferdinandusa goudotiana foram obtidos das folhas coletadas na Reserva Particular do Patrimônio Natural Cachoeira da Onça, no município de Presidente Figueiredo, na rodovia BR-174 (AM), este material faz parte do levantamento da flora da RPPN (Baze et al., 2003) e não possui exsicata depositada em herbário. Os extratos diclorometânicos e metanólicos das demais espécies foram preparados a partir das folhas coletadas na Reserva Florestal Adolfo Ducke, pertencente ao Instituto Nacional de Pesquisas da Amazônia (INPA), localizada 26 km a NE de Manaus, na rodovia AM-010. As exsicatas estão depositadas no herbário do mesmo Instituto, Duroia saccifera (222502), Ferdinandusa hirsuta (222503), F. paraensis (222504), Ferdinandusa sp. (222505), Palicourea corymbifera (222506) e P. guianensis (222507).

\section{Prospecção Fitoquímica}

Cada extrato obtido foi submetido a testes de prospecção fitoquímica para verificar a presença das seguintes classes de compostos: alcalóides, antocianidinas, antocianinas, antranóis, auronas, catequina (taninos catéquicos), chalconas, esteróides, fenóis simples, flavonas, flavonóis, flavanonas, flavanonóis, leucoantocianidinas, quinonas, taninos condensados, taninos hidrolisáveis, triterpenóides e xantonas, conforme os ensaios descritos por Mattos (1997).

\section{Leach.}

Atividade Citotóxica sobre Artemia salina

O bioensaio envolvendo Artemia salina consiste em avaliar a exposição de um determinado extrato ou composto frente a esse crustáceo. Foram pesados $10 \mathrm{mg}$ de cistos de Artemia salina adquiridos no comércio local, os quais foram adicionados em solução aquosa de sal marinho sintético (38 g L-1), sob iluminação artificial com lâmpada fluorescente e estado de saturação de oxigênio conseguido com auxílio de uma bomba de ar conectada ao béquer, este foi recoberto com parafilme durante $48 \mathrm{~h}$ para a eclosão das larvas. Após esse período, grupos de 10 larvas foram transferidas para poços de uma microplaca. A sala onde foi realizado o teste foi mantida em temperatura constante de $25^{\circ} \mathrm{C}$. Foram pesados $30 \mathrm{mg}$ dos extratos diclorometânico e metanólico e solubilizados em DMSO. A concentração inicial foi de $30 \mathrm{mg} \mathrm{mL}^{-1}$ (Meyer et al.,1982; Mongelli et al., 1996). O efeito tóxico dos extratos foi testado pelo método de Meyer e colaboradores, 1982. Para cada extrato

Rev. Bras. PI. Med., Campinas, v.17, n.4, p.604-613, 2015. 
o teste foi realizado em triplicata numa concentração de $1000 \mu \mathrm{g} \mathrm{mL}^{-1}$. A atividade foi determinada pela porcentagem de mortalidade observada após 24 $\mathrm{h}$ de incubação. Os extratos que se mostraram mais ativos foram avaliados em concentrações menores para a obtenção da $\mathrm{CL}_{50}$. O ensaio contou com dois grupos controle, sendo um constituído da solução salina (larvas + solução salina) e outro com o solvente (larvas + solvente), nas mesmas concentrações dos extratos. A análise dos dados finais foi feita pelo método Probit (Finney, 1962) e os resultados expressos como Concentração Letal Média $\left(\mathrm{CL}_{50}\right)$, ou seja, a concentração que dizima metade de uma população.

\section{Atividade Antioxidante}

A atividade antioxidante foi avaliada qualitativamente através da borrifação da placa cromatográfica, eluída e seca, com uma solução $0,2 \%$ de DPPH em MeOH. (Cuendet et al., 1997).

A avaliação da atividade antioxidante também foi realizada através de dois métodos quantitativos medindo a capacidade de seqüestro (CS) de radicais com DPPH e com ABTS ${ }^{+}$. Os resultados obtidos foram expressos em equivalência com o ácido ascórbico, termo este adotado para expressar a razão da concentração da amostra pela concentração de ácido ascórbico. A concentração equivalente de ácido ascórbico foi obtida pela interpolação dos dados de variação de absorbância e concentração de ácido ascórbico na curva doseresposta para cada método. Estes dois termos são distintos e o significado do termo "equivalência com o ácido ascórbico" permite correlacionar a resposta obtida do extrato vegetal com a do antioxidante padrão e quanto mais próximo de 1,0 , mais semelhante é a capacidade antioxidante da amostra ao ácido ascórbico (Carvalho, 2006).

\section{Atividade Antioxidante - Preparação da Curva Dose-Resposta do Ácido Ascórbico}

Pesaram-se $10 \mathrm{mg}$ de ácido ascórbico e em seguida diluiu-se em $25 \mathrm{~mL}$ de metanol grau P.A. (para o ensaio com DPPH) e em $25 \mathrm{~mL}$ de água deionizada (para o ensaio com ABTS) resultando na solução de concentração de $400 \mu \mathrm{g} \mathrm{mL}^{-1}$.

O procedimento consistiu na adição de $1000 \mu \mathrm{L}$ do radical DPPH (para o ensaio com DPPH), ou $1000 \mu \mathrm{L}$ do cátion radical ABTS (para o ensaio com ABTS) em microtubos Eppendorf e em seguida adicionou-se 99 ( $1^{\circ}$ tubo); 97 ( $2^{\circ}$ tubo); 96 ( $3^{\circ}$ tubo); 95 ( $4^{\circ}$ tubo); 90 ( $5^{\circ}$ tubo); 86 ( $6^{\circ}$ tubo) e 82 ( $7^{\circ}$ tubo) $\mu \mathrm{L}$ de $\mathrm{MeOH}$ grau P.A. e completou-se com $1\left(1^{\circ}\right.$ tubo); 3 ( $2^{\circ}$ tubo); 4 ( $3^{\circ}$ tubo); 5 ( $4^{\circ}$ tubo); 10 ( $5^{\circ}$ tubo); 14 ( $6^{\circ}$ tubo) e 18 ( $7^{\circ}$ tubo) $\mu \mathrm{L}$ de ácido ascórbico. $\mathrm{O}$ preparo do branco consistiu em $1000 \mu \mathrm{L}$ do radical DPPH (para o ensaio com DPPH) ou $1000 \mu \mathrm{L}$ do cátion radical ABTS (para o ensaio com ABTS), com $100 \mu \mathrm{L}$ de $\mathrm{MeOH}$ grau P.A. A absorbância foi medida no espectrofotômetro (FEMTO $800 \mathrm{XI}$ ) em $517 \mathrm{~nm}$ após 20 minutos de reação para o ensaio com DPPH, e em $734 \mathrm{~nm}$ após 6 minutos de reação para o ensaio com ABTS (Figura 1).

A curva dose-resposta obtida, expressa a concentração final de ácido ascórbico em $\mu \mathrm{g} \mathrm{mL}^{-1} \mathrm{e}$ a variação de absorbância ( $\triangle A B S)$.

\section{Avaliação da Atividade Antioxidante}

A solução de DPPH foi preparada dissolvendo-se $3,0 \mathrm{mg}$ do reagente em $100 \mathrm{~mL}$ de $\mathrm{MeOH}$ grau P.A.

$O$ cátion radical $A B T S$ foi produzido por meio químico através da reação da solução de ABTS 3,64 mM (solução aquosa) e persulfato de potássio a $5 \mathrm{mM}$ na razão estequiométrica de 1:0,5. A solução de ABTS foi armazenada em frasco âmbar e mantida em temperatura ambiente. Após 16 horas, a solução de ABTS foi diluída com metanol para uma absorbância em torno de 1,0.

A reação do teste amostra foi iniciada pela adição de $1000 \mu \mathrm{L}$ da solução de DPPH (para o ensaio com DPPH) ou $1000 \mu \mathrm{L}$ da solução de ABTS (para o ensaio com ABTS), $12 \mu \mathrm{L}$ de $\mathrm{MeOH}$ e 88 $\mu \mathrm{L}$ de amostra $(0,5 \mathrm{mg} / \mathrm{mL})$. O preparo do branco consistiu em $1012 \mu \mathrm{L}$ de $\mathrm{MeOH}$ grau P.A. com 88 $\mu \mathrm{L}$ da amostra, enquanto o preparo do controle consistiu em $1100 \mu \mathrm{L}$ da solução de DPPH ou solução de ABTS. As leituras foram realizadas em dois tempos de reação com 20 minutos em 517 nm (para o ensaio com DPPH), e com 6 minutos em $734 \mathrm{~nm}$ (para o ensaio com ABTS), (Figura 1), no espectrofotômetro (FEMTO 800 XI), e a variação de absorbância $(\triangle A B S)$ foi obtida pela seguinte equação:

\section{ABS branco)}

$$
\triangle \mathrm{ABS}=\mathrm{ABS} \text { controle }-(\mathrm{ABS} \text { amostra }-
$$

Onde: $A B S$ controle= absorbância obtida do controle; ABS amostra= absorbância obtida da amostra; ABS branco = absorbância obtida do branco.

\section{Atividade Antifúngica}

Foram usadas cepas da coleção de fungos de interesse médico da Coordenação de Pesquisas em Ciências da Saúde do Instituto Nacional de Pesquisas da Amazônia. Os extratos foram testados para a verificação de atividade inibitória contra os fungos filamentosos dermatofíticos Epidermophyton floccosum, Microsporum canis 32905, M. gipseum 29/00, Trichophyton mentagrophytes ATCC 9533/03, T. rubrum ATCC 28189 e T. tonsurans $21 / 97$, os fungos filamentosos não dermatofíticos 


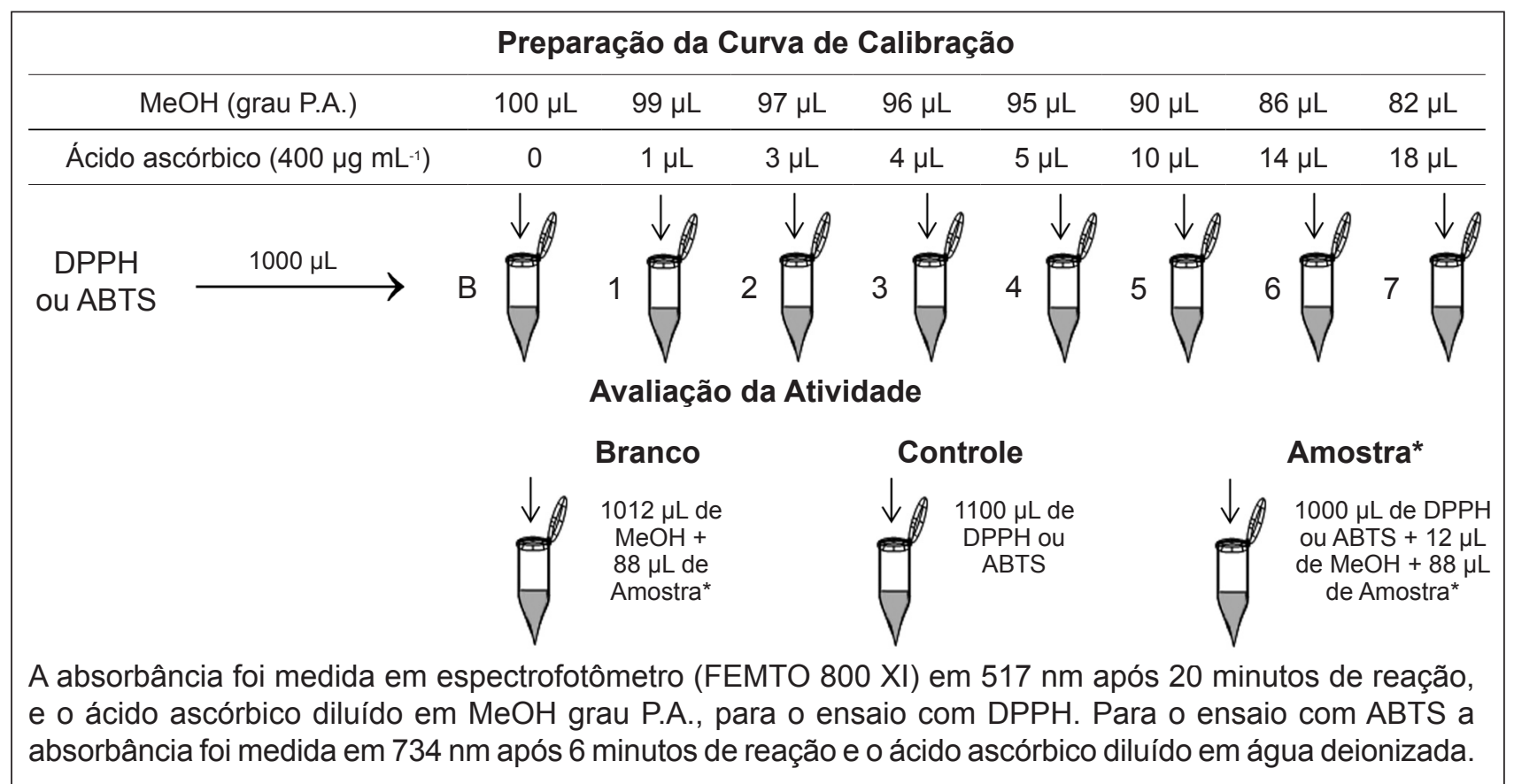

FIGURA 1. Ensaio de redução do radical livre DPPH e descoloração do cátion radical ABTS.

Cladosporium cladosporioides, C. sphaerospermum, Fusarium U. 662/06 e Scytalidium U. 661/06 e leveduriformes Candida albicans ATCC 3632 e C. albicans U. 5/99.

\section{Atividade Antifúngica - Preparação do Inóculo Fúngico}

As cepas fúngicas foram sub-cultivadas em meio de Sabouraud com extrato de levedura à $29^{\circ} \mathrm{C}$. Após o pleno desenvolvimento das colônias foi retirada uma parte da colônia e transferida para tubo de ensaio adicionando-se $2 \mathrm{~mL}$ de água destilada, obtendo-se assim, a suspensão fúngica segundo as recomendações do National Committee for Clinical Laboratory Standards (NCCLS, M38-P). A concentração dessa suspensão foi ajustada em espectrofotômetro a $530 \mathrm{~nm}$, com transmitância de 65 a 70, correspondendo a $10^{6}$ UFC mL-1 (Fernández-Torrez et al., 2001; Pujol et al., 2002).

\section{Avaliação da Atividade Antifúngica pelo Método de Difusão por Cavidade Placa}

A metodologia aplicada foi aquela que utiliza a difusão por cavidade-placa (Método de KirbyBauer), recomendado internacionalmente para a determinação da sensibilidade, através da difusão em ágar (Grove \& Randall, 1955). Esse método é utilizado para a determinação da atividade inibitória dos diferentes agentes antifúngicos (extratos). Utilizou-se meio de ágar Sabouraud sólido em placas de Petri, já com o inoculo fúngico, nas quais escavaram-se poços onde foram inoculados os extratos vegetais a $29{ }^{\circ} \mathrm{C}$, por 10 dias, quando se procedeu à leitura dos halos de inibição. Os extratos foram testados na concentração de 0,1 $\mathrm{mg} \mu \mathrm{L}^{-1}$, os solventes utilizados foram o DMSO (dimetilssulfóxido) para os extratos hidrofóbicos e água destilada para os extratos hidrofílicos.

\section{RESULTADOS E DISCUSSÃO Prospecção Fitoquímica}

$\mathrm{Na}$ Tabela 1 são apresentados os resultados da prospecção fitoquímica dos extratos analisados, de acordo com os ensaios descritos por Mattos (1997). Observou-se que os extratos diclorometânicos e metanólicos apresentaram praticamente a mesma classe de substâncias (alcaloides, esteroides, fenóis simples, flavanonas e flavanonóis), apresentando maior intensidade no extrato metanólico, exceto para Ferdinandusa hirsuta e Palicourea corymbifera. Observou-se ainda a presença de triterpenóides nos extratos metanólico de Duroia saccifera e Ferdinandusa sp., e também leucoantocianidinas no extrato diclorometânico de Palicourea corymbifera. A presença de alcalóides em todos os extratos condiz com a quimiossistemática da família Rubiaceae, antraquinonas não foram encontradas, e não foram realizados testes para iridoides, outros marcadores quimiotaxonômicos dessa família segundo Bolzani et al. (2001). É importante ressaltar que esses são ensaios preliminares e que indicam a possibilidade ou não de se encontrar determinadas classes de substâncias nessas espécies, sendo necessário estudos químicos posteriores para melhor compreensão das mesmas. 
TABELA 1. Resultado da prospecção fitoquímica dos extratos brutos obtidos das folhas das diferentes espécies.

\begin{tabular}{|c|c|c|c|c|c|c|c|c|c|c|c|c|c|c|}
\hline $\begin{array}{l}\text { Classe de } \\
\text { Substâncias }\end{array}$ & EDDs & EMDs & EDFg & EMFg & EDFh & EMFh & EDFp & EMFp & EDFsp. & EMFsp. & EDPc & EMPc & EDPg & EMPg \\
\hline Alcalóides & + & ++ & ++ & +++ & + & ++ & + & ++ & + & ++ & + & ++ & + & ++ \\
\hline Antocianidinas & - & - & - & - & - & - & - & - & - & - & - & - & - & - \\
\hline Antocianinas & - & - & - & - & - & - & - & - & - & - & - & - & - & - \\
\hline Antranóis & - & - & - & - & - & - & - & - & - & - & - & - & - & - \\
\hline Auronas & - & - & - & - & - & - & & - & - & - & - & - & - & - \\
\hline Catequina & - & - & - & - & - & - & - & - & - & - & - & - & - & - \\
\hline Chalconas & - & - & - & - & - & - & - & - & - & - & - & - & - & - \\
\hline Esteróides & S & - & ++ & ++ & ++ & +++ & - & ++ & +++ & - & +++ & +++ & +++ & +++ \\
\hline Fenóis simples & ++ & ++ & ++ & ++ & & & & +++ & +++ & +++ & - & - & - & - \\
\hline Flavonas & - & - & - & - & - & - & - & - & - & - & - & - & - & - \\
\hline Flavonóis & - & - & - & - & - & - & - & - & - & - & - & - & - & - \\
\hline Flavanonas & ++ & +++ & ++ & +++ & ++ & - & ++ & +++ & +++ & +++ & - & - & ++ & ++ \\
\hline Flavanonóis & ++ & +++ & ++ & +++ & ++ & - & ++ & +++ & +++ & +++ & - & - & ++ & ++ \\
\hline Leucoantocianidina & - & - & - & - & - & - & - & - & - & - & + & - & - & - \\
\hline Quinonas & - & - & S & + & - & - & - & + & ++ & - & S & - & - & - \\
\hline Taninos condensados & - & - & - & - & - & - & - & - & - & - & - & - & - & - \\
\hline Taninos hidrolisáveis & - & - & - & - & - & - & - & - & - & - & - & - & - & - \\
\hline Triterpenóides & - & ++ & - & - & - & - & - & - & - & + & - & - & - & - \\
\hline Xantonas & - & - & - & - & - & - & - & - & - & - & - & - & - & - \\
\hline
\end{tabular}

Convenção: Forte: +++ Médio: ++ Fraco: + Suspeito: S Ausente: -

\section{Leach.}

Atividade Citotóxica sobre Artemia salina

$\mathrm{Na}$ avaliação de extratos vegetais por Artemia salina, resultados de $\mathrm{CL}_{50}$ menores que $1000 \mu \mathrm{g} \mathrm{mL} \mathrm{m}^{-1}$ são considerados bioativos (Meyer et al., 1982). Considerou-se baixa toxicidade quando a concentração letal $\left(\mathrm{CL}_{50}\right)$ foi superior a $500 \mu \mathrm{g}$ $\mathrm{mL}^{-1}$; moderada para $\mathrm{CL}_{50}$ entre 100 a $500 \mu \mathrm{g} \mathrm{mL}^{-1} \mathrm{e}$ muito tóxico quando a $\mathrm{CL}_{50}$ foi inferior $100 \mu \mathrm{g} \mathrm{mL}-1$. A família Rubiaceae é conhecida por apresentar plantas que contêm substâncias tóxicas de diversas estruturas. Os resultados obtidos indicam que o gênero Ferdinandusa mostra grande potencial de toxicidade (Tabela 2). Comparando-se os valores de $\mathrm{CL}_{50}$, podemos observar um grande potencial de toxicidade no extrato metanólico de $F$. goudotiana, que mostrou-se tóxico até uma concentração de 5 $\mu \mathrm{g} \mathrm{mL}-1$, seguido do extrato metanólico de $F$. hirsuta $\left(\mathrm{CL}_{50}=67,91 \mu \mathrm{g} \mathrm{mL}^{-1}\right)$. Os extratos diclorometânicos de $F$. goudotiana $\left(\mathrm{CL}_{50}=376,11 \mu \mathrm{g} \mathrm{mL}-1\right)$, F. hirsuta $\left(\mathrm{CL}_{50}=174,17 \mu \mathrm{g} \mathrm{mL-1}\right)$ e F. paraensis $\left(\mathrm{CL}_{50}=\right.$ $158,61 \mu \mathrm{g} \mathrm{mL}^{-1}$ ) apresentaram atividade moderada. O extrato metanólico de F. paraensis apresentou baixa atividade, enquanto que os extratos de $D$. saccifera, Ferdinandusa SP, Palicourea corymbifera e P. guianensis não apresentaram toxicidade na concentração testada.

Uma vez que o ensaio de citotoxicidade sobre Artemia salina é o primeiro ensaio na busca de substâncias com atividade antitumoral (Siqueira et al., 2001), esses extratos deverão ser submetidos a outros ensaios antitumorais para avaliar o seu potencial. Assim como deverão ser avaliados em ensaios pré-clínicos em animais a fim de determinar a sua toxicidade. Os resultados obtidos para as espécies de Palicourea estão de acordo com Quignard e colaboradores, 2003, aonde foi constatado que essas mesmas espécies não eram tóxicas para Artemia franciscana.

O potencial tóxico observado no extratos de Ferdinandusa goudotiana, F. hirsuta e F. paraensis mostram a importância de estudos químicos posteriores para este gênero, uma vez que este estudo é o primeiro registrado na literatura consultada, até o presente momento para espécies deste gênero. 
TABELA 2. Resultados do ensaio de toxicidade sobre Artemia salina.

\begin{tabular}{|c|c|c|}
\hline Espécie & Extrato* & $\mathrm{CL}_{50}\left(\mu \mathrm{g} \mathrm{mL}^{-1}\right)$ \\
\hline \multirow{2}{*}{ D. saccifera } & DCM & \multirow{2}{*}{$>1000$} \\
\hline & $\mathrm{MeOH}$ & \\
\hline \multirow{2}{*}{ Ferdinandusa goudotiana } & DCM & $376,11 \pm 2,51$ \\
\hline & $\mathrm{MeOH}$ & $<5$ \\
\hline \multirow{2}{*}{ F. hirsuta } & DCM & $174,17 \pm 2,26$ \\
\hline & $\mathrm{MeOH}$ & $67,91 \pm 1,81$ \\
\hline \multirow{2}{*}{ F. paraensis } & DCM & $158,61 \pm 2,22$ \\
\hline & $\mathrm{MeOH}$ & $879,04 \pm 2,96$ \\
\hline \multirow{2}{*}{ Ferdinandusa sp. } & DCM & \multirow{2}{*}{$>1000$} \\
\hline & $\mathrm{MeOH}$ & \\
\hline \multirow{2}{*}{ Palicourea corymbifera } & DCM & \multirow{2}{*}{$>1000$} \\
\hline & $\mathrm{MeOH}$ & \\
\hline \multirow{2}{*}{ P. guianensis } & DCM & \multirow{2}{*}{$>1000$} \\
\hline & $\mathrm{MeOH}$ & \\
\hline
\end{tabular}

* Legenda: DCM = extrato diclorometânico; $\mathrm{MeOH}$ = extrato metanólico.

\section{Atividade Antioxidante - Ensaio Qualitativo}

O sistema de eluição utilizado foi diclorometano/metanol na proporção 9:1 para os extratos diclometânicos, e diclorometano/metanol na proporção 85:15 para os extratos metanólicos de Duroia saccifera (Ds), Ferdinandusa goudotiana $(F g)$, F. hirsuta (Fh), Ferdinandusa sp. (Fsp), Palicourea corymbifera $(P c)$ e $P$. guianensis $(P g)$. Após a borrifação das placas cromatográficas, previamente eluídas e secas, com a solução $0,2 \%$ de DPPH em $\mathrm{MeOH}$ (de coloração púrpura), o surgimento de bandas amarelas (Figura 2), mostra o potencial antioxidante dos extratos vegetais. Os extratos metanólicos mostraram bandas com forte intensidade da coloração amarela, com destaque para banda em $F g$ com $\mathrm{Rf}=0,46$ que apresentou maior intensidade. Os extratos diclorometânicos apresentaram coloração bem menos intensa, um indicativo de fraca atividade, esses resultados apresentaram boa correlação com os resultados da prospecção fitoquímica já apresentada na Tabela 1 que indica a presença dos flavanóides em maior quantidade nos extratos metanólicos, e pode-se inferir que a atividade antioxidante apresentada está relacionada à esta classe de substâncias já reconhecida por suas propriedades antioxidantes (Zuanazzi \& Montanha, 2003). As intensidades variadas para os diversos extratos sugerem que as espécies possuem diferentes características químicas e que os componentes mais polares presentes nos extratos metanólicos contribuíram para o aumento da atividade. Antioxidantes de variados extratos vegetais não atuam do mesmo modo e podem ser efetivos contra diferentes radicais livres (Choi et al., 2002).

\section{Atividade Antioxidante - Ensaios Quantitativos}

Os ensaios foram realizados no mesmo dia para diminuir as possibilidades de interferências nas condições de análise. As amostras foram preparadas a partir da solubilização dos extratos na concentração de $0,4 \mathrm{mg} \mathrm{mL}^{-1} \mathrm{em}$ metanol P.A.

O mecanismo de ação dos dois ensaios é baseado no sequestro de radicais livres e ao adicionar o provável antioxidante contido no extrato vegetal ocorre perda do radical cromógeno evidenciado através da redução de absorbância em determinado comprimento de onda. As diminuições foram correlacionadas em uma curva dose-resposta de um antioxidante padrão (Figura 3), o qual neste presente trabalho foi o ácido ascórbico, que é um antioxidante endógeno e apresenta atividade biológica. Foi considerado o antioxidante de referência deste método por expressar não somente dados comparativos e sim uma equivalência com antioxidante de atuação no organismo. Logo, os resultados são expressos comparativamente com a equivalência ao ácido ascórbico e os que aproximarem de 1,0 são considerados potentes 
Placas cromatográficas após eluição
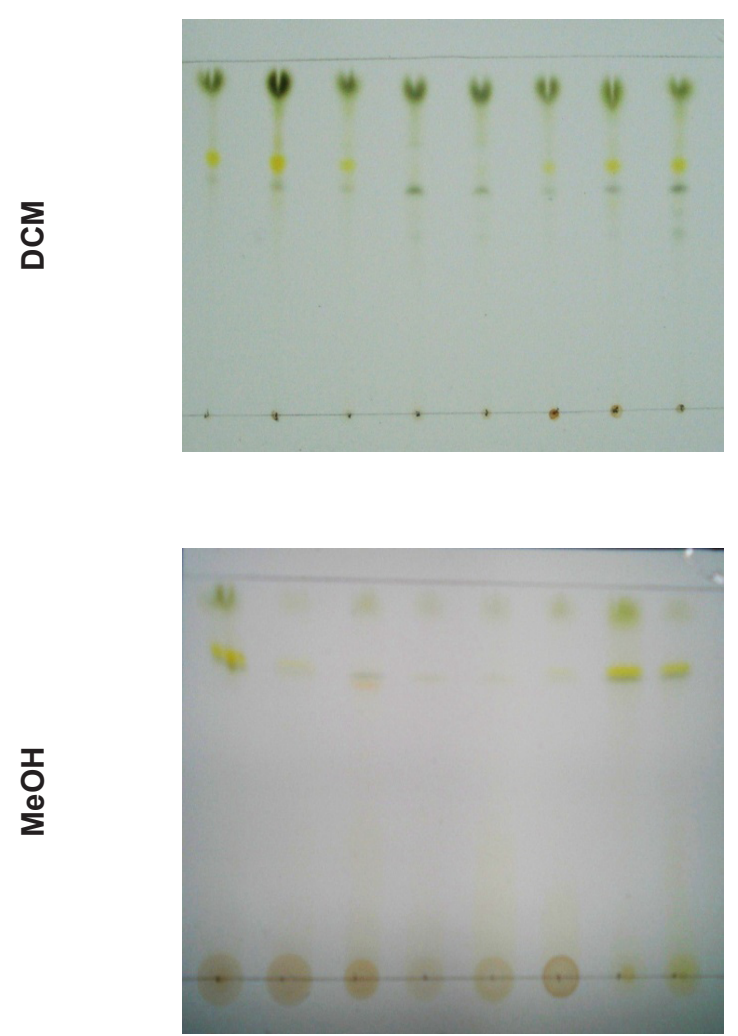

Ds Fg Fh Fp Fsp Pc Pg
Placas cromatográficas após borrifação com DPPH
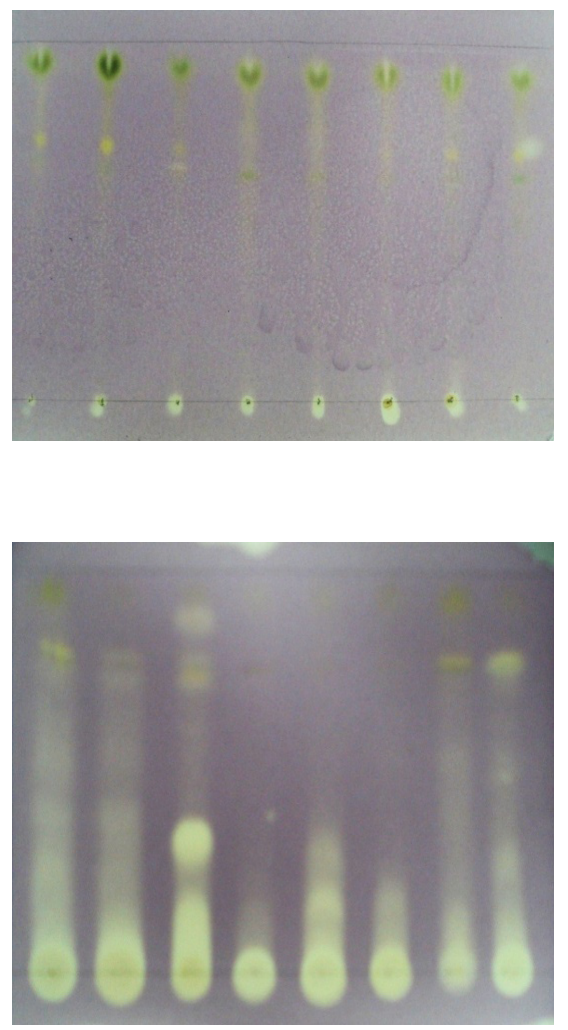

Ds Fg Fh Fp Fsp Pc Pg

FIGURA 2. Avaliação qualitativa da atividade antioxidante dos extratos.

antioxidantes (Carvalho, 2006).

Os resultados para os ensaios de redução do radical livre DPPH e de descoloração do cátion radical ABTS estão mostrados na Tabela 3.

Assim como no ensaio qualitativo, os extratos metanólicos mostraram-se mais eficientes em sequestrar os radicais livres DPPH e ABTS. Observou-se boa correlação entre os resultados obtidos para os dois ensaios, de redução do DPPH e o de descoloração do cátion radical ABTS.

$A$ atividade antioxidante desses extratos vegetais deve-se provavelmente à presença entre seus constituintes químicos de substâncias antioxidantes como os flavonóides, do mesmo modo pode-se sugerir que a atividade está relacionada ao efeito sinérgico das diferentes substâncias. As espécies estudadas são promissoras para maiores investigações posteriores sobre suas propriedades antioxidantes e possibilidades de aplicações, como por exemplo, a prevenção do estresse oxidativo no organismo humano causado por espécies reativas de oxigênio ou nitrogênio (ERO ou ERN) provenientes do meio ambiente ou gerada pelo próprio organismo, ou até mesmo para produção de antioxidantes alimentícios naturais em substituição aos sintéticos, o que é um a forte tendência, visto que as pesquisas têm demonstrado a possibilidade dos sintéticos apresentarem algum efeito tóxico (Barreiros et al., 2006; Ramalho \& Jorge, 2006).

\section{Atividade Antifúngica}

Atividade antifúngica e antibacteriana têm sido relatada na literatura para extratos de espécies de Rubiaceae (Agripino et al., 2004; Locher et al., 1995),

Os resultados dessa avaliação indicaram a presença de princípios antifúngicos nas espécies F. hirsuta e F. paraensis (Tabela 4), contra quatro dos fungos testados: Epidermophyton floccosum, Microsporum canis 32905, Trichophyton mentagrophytesATCC 9533/03 e T. rubrumATCC 28189. O extrato diclorometânico de F. hirsuta apresentou os melhores resultados, pois mostrouse ativo contra esses quatro fungos, seguido pelo extrato diclorometânico de F. paraensis ativo contra três fungos, só não contra Epidermophyton floccosum. Os extratos metanólicos mostraramse menos ativos, o de F. hirsuta foi ativo contra 


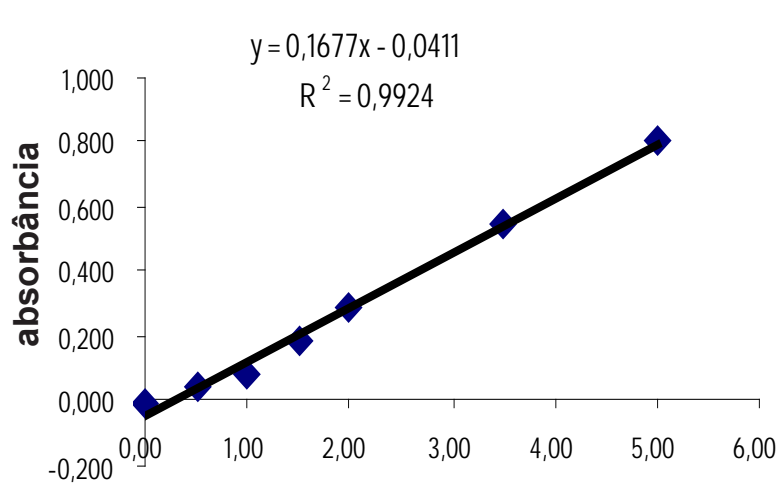

Concentração de Ác. Ascórbico

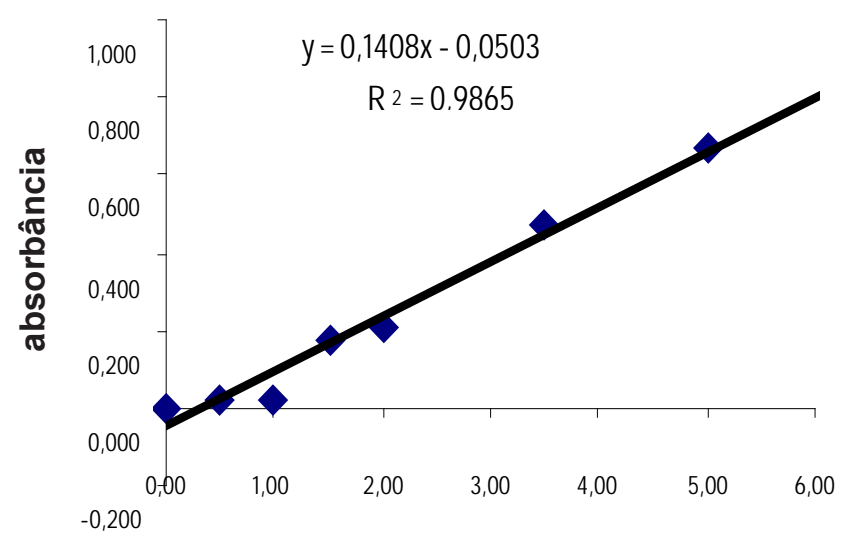

Concentracão de Ác. Ascórbico

FIGURA 3. Curvas dose-resposta do antioxidante padrão, o ácido ascórbico.

TABELA 3. Resultados da avaliação antioxidante nos extratos vegetais para os ensaios de redução do DPPH e para o ensaio de descoloração do cátion radical ABTS.

\begin{tabular}{|c|c|c|c|}
\hline \multirow{3}{*}{ Espécie } & \multirow{3}{*}{ Extrato } & \multicolumn{2}{|c|}{ Equivalência com o ác. ascórbico } \\
\hline & & DPPH & ABTS \\
\hline & & $20 \mathrm{~min}$ & $6 \mathrm{~min}$ \\
\hline \multirow{2}{*}{ D. saccifera } & DCM & $26,6 \pm 0,3$ & $19,2 \pm 0,4$ \\
\hline & $\mathrm{MeOH}$ & $7,3 \pm 0,1$ & 6,7 \\
\hline \multirow{2}{*}{ Ferdinandusa goudotiana } & DCM & $26,9 \pm 0,2$ & $12,5 \pm 0,1$ \\
\hline & $\mathrm{MeOH}$ & $5,4 \pm 0,1$ & 7,2 \\
\hline \multirow{2}{*}{ F. hirsuta } & DCM & $30,5 \pm 1,4$ & $33,9 \pm 0,7$ \\
\hline & $\mathrm{MeOH}$ & $6,0 \pm 0,1$ & 7,7 \\
\hline \multirow{2}{*}{ F. paraensis } & DCM & $32,9 \pm 0,8$ & $30,4 \pm 4,1$ \\
\hline & $\mathrm{MeOH}$ & 5,6 & 7,9 \\
\hline \multirow{2}{*}{ Ferdinandusa sp. } & DCM & 5,7 & $7,7 \pm 0,1$ \\
\hline & $\mathrm{MeOH}$ & 5,3 & 8,6 \\
\hline \multirow{2}{*}{ Palicourea corymbifera } & DCM & $28,9 \pm 0,4$ & $12,4 \pm 0,1$ \\
\hline & $\mathrm{MeOH}$ & $20,2 \pm 0,1$ & 7,2 \\
\hline \multirow{2}{*}{ P. guianensis } & DCM & $27,7 \pm 0,2$ & $12,6 \pm 0,1$ \\
\hline & $\mathrm{MeOH}$ & $11,7 \pm 0,2$ & 9,0 \\
\hline
\end{tabular}

Trichophyton mentagrophytes ATCC 9533/03 e T. rubrum ATCC 28189 e o de F. paraensis indicou atividade somente contra T. rubrum ATCC 28189. Os demais extratos não apresentaram atividade para nenhum dos fungos testados, na concentração e condições avaliadas. A ausência de atividade de todos os extratos frente aos fungos não dermatofíticos (7 a 10) e leveduriformes (11 e 12) denota, que esses outros fungos mostraram-se mais resistentes que os dermatofíticos testados.

O diâmetro dos halos de inibição correspondentes podem ser observados na figura.

Os resultados obtidos no estudo apontam para o grande potencial de atividades biológicas nas espécies de Ferdinandusa que apresentaram bons resultados em todas as atividades avaliadas. Conforme já mencionado, as espécies estudadas apresentam pouco ou nenhum relato para as 
TABELA 4. Atividade inibitória dos extratos contra os fungos.

\begin{tabular}{|c|c|c|c|c|c|c|c|c|c|c|c|c|}
\hline \multirow{3}{*}{ Extrato } & \multicolumn{12}{|c|}{ Fungos* (diâmetro do halo de inibição em cm) } \\
\hline & \multicolumn{5}{|c|}{ Filamentosos dermatofíticos } & \multicolumn{5}{|c|}{ Filamentosos não dermatofíticos } & \multicolumn{2}{|c|}{ Levedu-riformes } \\
\hline & 1 & 2 & 3 & 4 & 5 & 6 & 7 & 8 & 9 & 10 & 11 & 12 \\
\hline EDDs & - & - & - & - & - & - & - & - & - & - & - & - \\
\hline EMDs & - & - & - & - & - & - & - & - & - & - & - & - \\
\hline EDFg & - & - & - & - & - & - & - & - & - & - & - & - \\
\hline EMFg & - & - & - & - & - & - & - & - & - & - & - & - \\
\hline EDFh & 1,8 & 1,5 & - & 1,4 & 0,8 & - & - & - & - & - & - & - \\
\hline EMFh & - & - & - & 0,8 & 0,7 & - & - & - & - & - & - & - \\
\hline EDFp & - & 1,7 & - & 1,5 & 1,0 & - & - & - & - & - & - & - \\
\hline EMFp & - & - & - & - & 0,5 & - & - & - & - & - & - & - \\
\hline EDFsp. & - & - & - & - & - & - & - & - & - & - & - & - \\
\hline EMFsp. & - & - & - & - & - & - & - & - & - & - & - & - \\
\hline EDPc & - & - & - & - & - & - & - & - & - & - & - & - \\
\hline EMPc & - & - & - & - & - & - & - & - & - & - & - & - \\
\hline EDPg & - & - & - & - & - & - & - & - & - & - & - & - \\
\hline $\mathrm{EMPg}$ & - & - & - & - & - & - & - & - & - & - & - & - \\
\hline
\end{tabular}

- : indica ausência de atividade antifúngica

*Fungos: 1. Epidermophyton floccosum; 2. Microsporum canis 32905; 3. M. gipseum 29/00; 4. Trichophyton mentagrophytes ATCC 9533/03; 5. T. rubrum ATCC 28189; 6. T. tonsurans 21/97; 7. Cladosporium cladosporioides; 8. C. sphaerospermum; 9. Fusarium U. 662/06; 10. Scytalidium U. 661/06; 11. Candida albicans ATCC 3632; 12. C. albicans U. 5/99.

atividades testadas, na literatura consultada. Apesar de preliminares para uma comprovação dos potenciais antioxidantes, antifúngicos e citotóxicos, os resultados apresentam-se animadores e criam possibilidades para futuras investigações relacionadas à estrutura e atividade dos componentes dos extratos com atividade.

\section{AGRADECIMENTOS}

Aos botânicos Carlos Alberto Cid Ferreira e lêda Leão do Amaral, do Departamento de Botânica, do Instituto Nacional de Pesquisas da Amazônia pela identificação das espécies. MESQUITA, D. W. $\mathrm{O}$. agradece ao CNPq pela bolsa de mestrado e auxílio financeiro concedido, MESQUITA, A. S. S. e CURSINO, L. M. C. agradecem ao CNPq e INPA pelas bolsas de Iniciação Científica, SOUZA, E. S. agradece à FAPEAM pela bolsa de apoio técnico concedida, OLIVEIRA, A. C. agradece à FAPEAM pela bolsa de DCR e auxílio financeiro concedido e NUNEZ, C. V. agradece ao PPBio/INPA/CNPq/MCT pelos auxílios concedidos.

\section{REFERÊNCIAS}

AGRIPINO, D. G. et al. Screening of Brazilian plants for antimicrobial and DNA-Damging activities. I. Atlantic Rain Forest - Ecological Station Juréia - Itatins. Biota Neotropica, v. 4, n. 2, p.1-15, 2004.

ASSIS, J.C.S. R. et al. Analysis of the toxic potential of Palicourea corymbifera (Müll. Arg.) Standl. in laboratory animals. Research in Veterinary Science, v. 80, p.209-217, 2006.

BARREIROS, A. L. B. S.; et al. Estresse Oxidativo: Relação entre Geração de Espécies Reativas e Defesa do Organismo. Química Nova, v. 29, n. 1, p.113-123, 2006.

BAZE, A.; CORDEIRO, A. C.; BUENO, C. R.; PALÁCIO, C. A. S.; FERREIRA, C. A. C.; CRUZ, F. Reserva Ambiental da Cachoeira da Onça. Manaus-AM: Fundação Rede Amazônica/Instituto Nacional de Pesquisas da Amazônia, 2003. 106p.

BOLZANI, V. S. et al. Secondary Metabolites From Brazilian Rubiaceae: Chemotaxonomical and Biological Significance. Recent Research Developments in Phytochemistry, v. 5, p.19-31, 2001.

CARDOSO, C. L. et al. Indole monoterpene alkaloids from Chimarrhis turbinata DC Prodr.: a contribution to the chemotaxonomic studies of the Rubiaceae family. Revista Brasileira de Farmacognosia, v. 18, n.1, p. 
26-29, 2008.

CARVALHO, S. R. Otimização e Validação do Método ABTS e Avaliação das Atividades Antioxidantes de Extratos de Plantas Nativas da Amazônia. 2006. 70 p. Dissertação (Mestrado - Área de Concentração em Biotecnologia), Universidade do Estado do Amazonas, Manaus.

$\mathrm{CHOI}, \mathrm{C}$. W. et al. Antioxidant activity and free radical scavenging capacity between Korean medicinal plants and flvanoids by assay-guided comparison. Plant Science, v. 163, p.1161-1168, 2002.

CUENDET, M. et al. Iridoid glucosides with free radical scavenging properties from Fagraea blumei. Helvetica Chimica Acta, v. 80, p.1145-1152, 1997.

FERNÁNDEZ-TORREZ, B.; et al. In vitro activities of 10 antifungal drugs against 508 dermatophyte strains. Antimicrobiological Agents and Chemother, v. 45, n. 9, p.2524-2528. 2001.

FINNEY, D. J. Probit Analysis. London: Cambridge University Press, Cambridge, 1962. 333p.

GROVE, D. C.; RANDALL, W. A. Assay methods, Antibiotic Activity: A Laboratory Manual. New York: Medical Encyclopedia Inc., 1955. 192p.

LOCHER, C. P. et al. Anti-microbial activity and anticomplement activity of extracts obtained from selected Hawaiian medicinal plants. Journal of Ethnopharmacology, v. 49, p.23-32,1995.

MARTINS, D. et al. Triterpenes and the Antimycobacterial Activity of Duroia macrophylla Huber (Rubiaceae). BioMed Research International, v. 2013, p. 1-7, 2013.

MATTOS, F. J. A. Introdução à Fitoquímica Experimental. 2. ed. Fortaleza: Universidade Federal do Ceará, 1997. $141 \mathrm{p}$.

MEYER, B. N. et al. Brine Shrimp: A Conventional General Bioassay for Active Plant Constituents. Planta Medica. v. 45, p.31-34, 1982.

MONGELLI, E. et al. Screening of Argentine Medicinal Plants Using the Brine Shrimp Microwell Cytotoxicity Assay. Pharmaceutical Biology, v. 34, n. 4, p.249254, 1996.

PUJOL, I. et al. Use of the Colorimetric Microdilution Panel for Antifungal Susceptibility Testing of Dermatophytes. Journal of Clinical Microbiology, v. 40, n. 7, p.261821, 2002.

QUIGNARD, E.L.J. et al. Screening of plants found in Amazonas state for lethality towards brine shrimp. Acta Amazonica, v. 33, n. 1, p.93-104. 2003.

RAMALHO, V. C.; JORGE, N. Antioxidante utilizados em óleos, gorduras e alimentos gordurosos. Química Nova, v. 29 , n. 4 , p.755-760. 2006.

RIBEIRO, J. E. L. S.; HOPKINS, M. J. G.; VICENTINI, A.; SOTHERS, C. A.; COSTA, M. A. S.; BRITO, J. M.; SOUZA, M. A. D.; MARTINS, L. H. P.; LOHMANN, L. G.; ASSUNÇÃO, P. A. C. L.; PEREIRA, E. C.; SILVA, C. F.; MESQUITA, M. R.; PROCÓPIO, L. C. Flora da Reserva Ducke Guia de Identificação das plantas vasculares de uma floresta de terra-firme na Amazônia Central. Manaus - AM: INPA, DFID (Departament for International Development), 1999. 816p.

SIQUEIRA, J. M. et al. Estudo Fitoquímico das cascas do caule de Duguetia glabriuscula - Annonaceae, biomonitorado pelo ensaio de toxicidade frente a Artemia salina Leach. Química Nova, v. 24, n. 2, p.185187, 2001.

SOUZA, R. K. D. et al. Aspectos etnobotânicos, fitoquímicos e farmacológicos de espécies de Rubiaceae no Brasil. Revista Cubana de Plantas Medicinales, v. 18, n.1, p. 140-156, 2013.

VALENTE, L. M. M. et al. Kaempferitrin from Uncaria guianensis (Rubiaceae) and its potential as a chemical marker for the species. Journal of the Brazilian Chemical Society, v. 20, n. 6, p. 1041-1045, 2009.

VIEIRA, R. C. et al. Anatomical and Chemical Analyses of Leaf Secretory Cavities of Rustia Formosa (Rubiaceae). American Journal of Botany, v. 88, n. 12, p. 21512156, 2001.

ZUANAZZI, J. A. S.; MONTANHA, J. A. Flavonóides. In: SIMÕES, C. M. O.; SCHENKEL, E. P.; GOSMANM, G.; MELLO, J. C. P.; MENTZ, L. A.; PETROVICK, P. R. Farmacognosia: da Planta ao Medicamento. 5 ed. Porto Alegre/Florianópolis: Ed. da UFRGS/Ed. da UFSC, 2003 p. 578-614. 